\title{
Abundancia de carbono en microorganismos en suelos de estepa de Santa Cruz. Su alteración en función a la disponibilidad de humedad
}

\author{
Abundance of carbon in microorganisms in Santa Cruz steppe soils. Its alteration \\ depending on the availability of humidity
}

\author{
Guillermo Toledo ${ }^{1}$, Verónica Gargaglione ${ }^{1,2,3}$, Pablo Peri ${ }^{1,2,3}$ \\ 1)ICASUR/Ciencias Exactas y Naturales/UARG/UNPA,2)INTA, 3)CONICET
}

Recibido: 15/02/2019. Aceptado: 05/07/2019

\begin{abstract}
RESUMEN
El carbono de la biomasa microbiana (C-BM) del suelo es un bioindicador que tiene el potencial de dejar en evidencia rápidamente los cambios en el suelo de los ecosistemas, y está condicionada entre otros factores por la humedad disponible en el suelo. El presente trabajo tiene como objetivo evaluar como varía el C-BM en función de la cantidad de humedad que disponible en suelos de la Estepa Magallánica Seca de Santa Cruz, Argentina. El ensayo consistió en 3 tratamientos: a) Riego, con un aumento del 54\% en las precipitaciones respecto a la media anual de precipitaciones en la zona, b) Sequía, con una disminución del $54 \%$ en las precipitaciones respecto a la media anual en la zona, c) Control, sin variaciones en la precipitación, se utilizó como testigo. Se estimó el carbono contenido en los microorganismos del suelo mediante el método de fumigación-extracción. El suelo que presentó mayor contenido de C-MB fue testigo con 466,15 $( \pm 27,2) \mu \mathrm{g}$ de $\mathrm{C} / \mathrm{g}$ suelo seco, comparándolo con el suelo sometido a sequía que tuvo 394,08 $( \pm 29,8) \mu \mathrm{g}$ de C/g suelo seco. Por último, el suelo con riego presentó $462( \pm 29,6) \mu \mathrm{g}$ de C/g suelo seco. Estas diferencias no fueron significativas en este primer año de medición.
\end{abstract}

Palabras clave: Carbono microbiano; microorganismos; suelo; riego; sequía.

\begin{abstract}
Microbial carbón biomass (MBC) from soil is a bio-indicator that has the potential to show changes in the soil of ecosystems, it is conditioned by humidity. The objective of this research was to evaluate variation in MBC depending on water available on the ground from dry magallanic steppe in Santa Cruz, Argentina. The research consisted of three treatments: a) irrigation, 54\% increase in precipitation compared to the annual average in the area, b) drought, $54 \%$ decrease in precipitation compared to the annual average in the area, c) Control, without variations in precipitation, used as reference soil. MBC was estimated by the fumigation- extraction method. Reference soil had most MBC content, it was 466,15 $( \pm 27,2)$ $\mu \mathrm{g} \mathrm{C/g}$ dry soil, as compared to soil subject to drought, wich showed the lowest MBC content, it was $394,08( \pm 29,8) \mu \mathrm{g} \mathrm{C} / \mathrm{g}$ dry soil. The soil under irrigation showed $462( \pm 29,6) \mu \mathrm{g} \mathrm{C} / \mathrm{g}$ dry soil. These differences were not significant in this first year of measurement
\end{abstract}

Key words: Microbial carbon; microorganisms; soil; drought; irrigation. 


\section{Problemática actual:}

\section{INTRODUCCIÓN}

En Patagonia Sur, donde los pastizales son ampliamente utilizados para la cría de ganado, una herramienta a utilizar para tratar de aumentar la productividad del sistema puede ser mediante la implementación de alguna superficie bajo riego. Por otra parte, algunas predicciones de cambio climático indican que para la zona esteparia del Sur de Santa Cruz se prevé que la precipitación media anual se mantenga o se observe un leve incremento con respecto a los valores actuales (Kreps et al., 2012). En este contexto, conocer cómo es la biología de estos suelos en cuanto a carbono en los microorganismos y su respiración potencial, que da idea de la descomposición de la materia orgánica y por ende del ciclado de los nutrientes, puede servir de base para el entendimiento del funcionamiento de estos sistemas como así también para plantear diversos escenarios futuros relacionados a cambios climáticos en estos ambientes o a prácticas agrícolas de mejoramiento. Actualmente, en el sur de Patagonia existen escasos antecedentes de mediciones de contenido de $\mathrm{C}$ en biomasa microbiana en ambientes de pastizales y poco se sabe además de la respuesta del mismo a cambios en la disponibilidad de nutrientes y agua, por lo que esta beca pretende generar información complementaria sustancial para el entendimiento de estos ecosistemas australes en este aspecto.

\section{Marco Teórico:}

En los ecosistemas terrestres, la descomposición de la materia orgánica es un importante proceso mediado por microorganismos heterótrofos que utilizan al material orgánico muerto -o detritus- como hábitat y fuente de carbono (C) y energía. La descomposición es un proceso complejo por el cual la materia orgánica es degradada a partículas más pequeñas y a formas solubles de nutrientes que quedan disponibles para la absorción vegetal en parte, y otra parte queda inmovilizada en la biomasa microbiana. Este proceso de descomposición contribuye a la formación de la materia orgánica del suelo (Swift et al., 1979) y es una vía fundamental en el ciclado de nutrientes ya que la mayoría de los nutrientes disponibles del suelo derivan de la descomposición de la materia fresca de detritos vegetales y microbios asociados (Visser y Parkinson, 1992). Numerosos factores pueden influir en el proceso de descomposición, como la temperatura y humedad del ambiente, la composición de la comunidad microbiana y la cantidad y calidad del recurso a descomponer (Couteaux et al. 1995; Aerts 1997; Vázquez y Dávila 2008). Asimismo, el componente microbiológico puede servir como indicador del estado general del suelo, pues una alta actividad microbiana es asociada a un buen nivel de fertilidad y constituye un marcador biológico potencialmente útil para evaluar las perturbaciones que puedan presentarse. En este sentido, el $\mathrm{C}$ contenido en la biomasa microbiana es un componente lábil del pool de la materia orgánica del suelo y es considerado un buen indicador a corto plazo de los efectos de las distintas prácticas del manejo sobre las propiedades biológicas del suelo (Carter y Rennie, 1982; Campbell et al., 1991; Franzluebbers et al., 1994) ya que, por ejemplo, suelos disturbados usualmente contienen menores valores de biomasa microbiana que suelos de bosques y pastizales.

\section{Relación del presente trabajo con otras líneas de investigación en curso:}

El presente trabajo desarrollado se enmarca en el PI PI29/A 403-1 "Estudiando la biología del suelo en pastizales áridos del Sur de Santa Cruz: Respuesta de los microorganismos del suelo a distintos niveles de fertilización y riego", el cual busca generar información en cuanto 
a microorganismos en suelo de estepa seca del sur de Santa cruz y su respuesta a las condiciones ambientales.

\section{Importancia de los microorganismos en los ecosistemas}

La actividad de los microorganismos en el suelo es, no sólo, un factor clave en la fertilidad del suelo, sino que también lo es en la estabilidad y funcionamiento de los ecosistemas naturales y en los agroecosistemas (Trasar et al., 2000). Es importante destacar la relevancia de los microorganismos y de su actividad en la calidad del suelo y, por tanto, en su conservación y mantenimiento. Brookes (1995) describe que la actividad microbiana es un parámetro que responde rápidamente a los cambios provocados por el ambiente y las prácticas de manejo del suelo.

El componente biológico del suelo puede ser considerado como un "alerta" ante un posible perjuicio en el mismo, permitiéndose entonces reaccionar a tiempo antes de que pueda ocurrir un daño irreversible en el suelo (García Izquierdo et al., 2003). Es por ello, que el carbono contenido en la biomasa microbiana del suelo (C-BM) ha sido utilizado como un indicador de cambios ocurridos en el ambiente edáfico producto de la aplicación de diferentes sistemas de manejo agropecuario, dado que resulta ser un indicador del impacto del sistemas sobre los niveles de materia orgánica del suelo, y mucho más importante aún, sobre la calidad de esta materia orgánica como sustrato (Bending et al., 2000). La biomasa microbiana responde rápidamente a los cambios de manejo de uso de la tierra y proporciona una advertencia de una transición a otro estado que no está en equilibrio en el sistema (Jenkinson, 1988). Una disminución de los niveles de biomasa microbiana del suelo, acarrea problemas por afectar el ciclaje de nutrientes y su disponibilidad (Landgraf y Klose, 2002). Los nutrientes que puede contener un suelo se asocia con el C-BM, ya que la biomasa microbiana representa un 1-4 \% del carbono orgánico del suelo (Paul y Clark, 1989). Estas estimaciones son posibles gracias a que elementos como el carbono, el nitrógeno y el fósforo son esenciales en la conformación de la estructura celular (Anderson y Domsch, 1986; Sparling, 1985).

\section{Efecto del contenido de humedad del suelo sobre los microorganismos del suelo}

El contenido de humedad del suelo es un factor que afecta a los microorganismos, y muchas veces la capacidad de retención de agua del suelo está condicionada por las propiedades físicas, donde la estabilidad de los agregados del suelo se favorece por los exudados de los microorganismos que habitan en él (Edgerton et al., 1995). A su vez, un mayor contenido de humedad del suelo incrementa la descomposición de la materia orgánica del suelo por el carbono lábil de la biomasa microbiana (Fierrer y Schimel, 2003; Saetre y Stark, 2005).

Las predicciones de cambio climático indican que los años extremadamente secos tienden a ser más frecuentes en el futuro, modificándose también los patrones de precipitaciones (Houghton et al., 2001). Para la región de Patagonia Sur, se predice un aumento de las temperaturas acompañada de aumentos en la precipitación que van de sur a norte para la zona sur de la provincia de Santa Cruz (Kreps et al., 2012). Es por ello que poder observar de qué manera puede incidir el contenido de humedad del suelo sobre los microorganismos sería de interés para interpretar comportamientos a nivel de la comunidad microbiológica y su funcionalidad en estos ecosistemas patagónicos que presentan limitada disponibilidad de agua.

Las mejores condiciones de humedad en los suelos incrementan la biomasa microbiana y estimula a una mayor diversidad de microorganismos durante ese periodo, aparejado por una mayor funcionalidad de procesos microbiológicos del suelo (Kandeler y Bohn, 1996). Fierre y Schimel (2003) encontraron que la actividad microbiana representada por la biomasa 
microbiana se favorece con el mayor contenido de humedad en el suelo, lográndose obtener una correlación positiva. Vásquez y Dávila (2008) en su trabajo sobre los afectos de los factores abióticos sobre la actividad microbiana, encontró que contenidos de humedad mayores a $18 \%$ favorecen la actividad de los microorganismos cuando otros factores se mantienen constantes.

Los efectos de la sequía sobre los microorganismos del suelo fueron estudiados in situ en ecosistemas de Dinamarca y norte de Gales (Jensen et al., 2003), donde el tratamiento de sequía ("eliminación de la precipitación") redujo la biomasa microbiana en un $39 \%$ después de dos meses de ensayo. Las respuestas encontradas en estos dos ecosistemas se relacionan con mayor retención de agua producto del contenido de materia orgánica y textura del suelo. Sin embargo, Wardle (1998) detalla que hay tendencias contradictorias en el efecto que tiene la humedad del suelo y la producción de restos vegetales sobre la biomasa microbiana del suelo. Por ejemplo, Raghurbanshi (1994) encontró una relación negativa entre la humedad del suelo y la biomasa microbiana. Entre las causales de la sequía, Adu y Oades (1978) describieron que la biomasa microbiana disminuye durante el estrés hídrico de la temporada seca por efecto de la presión de turgencia a la que está sometida, y que la biomasa sobreviviente en la estación húmeda, favorece la mineralización del carbono lábil de la biomasa microbiana muerta con su consecuente aumento del carbono de la biomasa microbiana remanente del suelo. La menor actividad microbiana está asociada con el estrés hídrico en la temporada climática seca en las sabanas, en la cual gran parte de los microorganismos del suelo mueren o permanecen inactivos (Shing et al., 1989). Brock et al. (1984) menciona que la actividad microbiana después de un periodo de sequía, a pesar que se le agregue humedad al suelo ella no mostrara cambios dado que las células están lisadas y esa recuperación le llevará tiempo a la biota del suelo.

En muchos casos el contenido de humedad interactúa con la temperatura sobre las poblaciones microbianas y sus enzimas (Lavalle et al., 1993; Chapin et al., 2002). En climas templados la biomasa microbiana es máxima en primavera o verano y disminuye en invierno y esto se acentuará en las latitudes más altas (Cochran et al., 1989). Estudios encontraron una relación positiva de la tasa de respiración de los suelos, el contenido hídrico de los suelos y la temperatura, como resultado de la actividad biológica (Gupta y Singh, 1981; Chapin et al., 2002; Carmona et al., 2006).Este tipo de información es fundamental para lograr comprender la dinámica de estos ecosistemas y de qué manera se ven afectados los microorganismos en función de la disponibilidad de humedad en las capas superficiales del suelo. Sin embargo, no existen muchos estudios sobre esto en Patagonia sur, razón por la cual este trabajo puede aportar información al conocimiento que se tiene sobre la implicancia de las variaciones de humedad en suelo y de qué manera afecta esto a la abundancia de fauna microbiológica en suelos de estepa de Santa Cruz.

\section{Objetivos:}

- Evaluar el contenido de $\mathrm{C}$ de un pastizal natural de la estepa magallánica seca que se encuentra con clausura de pastoreo hace más de 20 años para poder establecer la línea de base en cuanto a la biología de estos suelos.

- Evaluar la respuesta de los microorganismos del suelo ante una sequía, que comprende que en tres años consecutivos caiga un 54\% menos de la precipitación media anual del sitio.

- Evaluar la respuesta de los microorganismos del suelo ante un aumento de las precipitaciones, que comprende que en tres años consecutivos caiga un 54\% más de la precipitación media anual del sitio. 


\section{Sitio de estudio:}

\section{METODOLOGÍA DE TRABAJO}

El estudio se realizó en el campo campo experimental Potrok Aike de la EEA-INTA Santa Cruz (51 $56^{\prime} 57^{\prime \prime} \mathrm{LS}$ y $\left.70^{\circ} 24^{\prime} 42^{\prime \prime} \mathrm{LO}\right)$, ubicado a $107 \mathrm{KM}$ en dirección SO de la localidad de Río Gallegos, dentro del área ecológica denominada Estepa Magallánica Seca (Oliva et al., 2001). La temperatura media anual es de $5,9^{\circ} \mathrm{C}$ y la precipitación median anual es de 220 $\mathrm{mm}$. La vegetación dominante corresponde a una estepa graminosa de Festuca gracillima principalmente (14\%) acompañada de pastos cortos (33\%) como ser Poa spiciformis, Carex andina y Ritidosperma virescens y algunos subarbustos (12\%). Los suelos en general son molisoles de textura arenosa. Las parcelas de presente ensayo fueron instaladas en una clausura de pastoreo que tiene alrededor de 20 años. El diseño corresponde a un diseño factorial en bloques, con tres repeticiones.

\section{Experimento hídrico}

Se colocaron estructuras fijas que reducen de forma pasiva un porcentaje de la precipitación mediante el uso de interceptores (Figura 1). En todos los casos, la cantidad total de la reducción o adición de las precipitaciones se cuantificó a través de datos recolectados sobre una base de registros de largo plazo, desde 1896 de Río Gallegos (cercano al lugar de estudio). Para poder determinar el nivel de la extremidad de la sequía hay una función matemática puesta a disposición en el sitio web de la Sequía-Net (www.drought-net.org). Para la media de $243,4 \mathrm{~mm}_{\text {año }}{ }^{-1}$ de precipitaciones registradas en el área de estudio se estimó los valores mínimos para el caso de la sequía extrema igual a 111,9 mm año ${ }^{-1}$ (reducción del 54\%). En el caso de suplementación de la precipitación, se aplicaron un total de 6 riegos programadas durante el año con el objetivo de lograr un aumento de la precipitación del 54\% del valor medio histórico, lo cual corresponde a una adición total de $131.4 \mathrm{~mm}$ año ${ }^{-1}$; todo esto esto se enmarca dentro del plan de trabajo del PI PI29/A 403-1 antes mencionado. De esta manera los tratamientos consistieron: 1) tratamiento de precipitación ambiental (Control, $243.4 \mathrm{~mm}$ año $\left.^{-1}\right)$, 2) tratamiento de sequía reducción del 54\% (111.9 mm año-1) y 3) un tratamiento de suplemento de precipitación del $54 \%\left(374.8 \mathrm{~mm} \mathrm{año}^{-1}\right)$. Cada tratamiento posee tres repeticiones (parcelas de $2 \times 2$ de $4 \mathrm{~m} 2$ ), distribuidas al azar en el área de estudio, en un diseño en bloques, como se mencionara anteriormente.
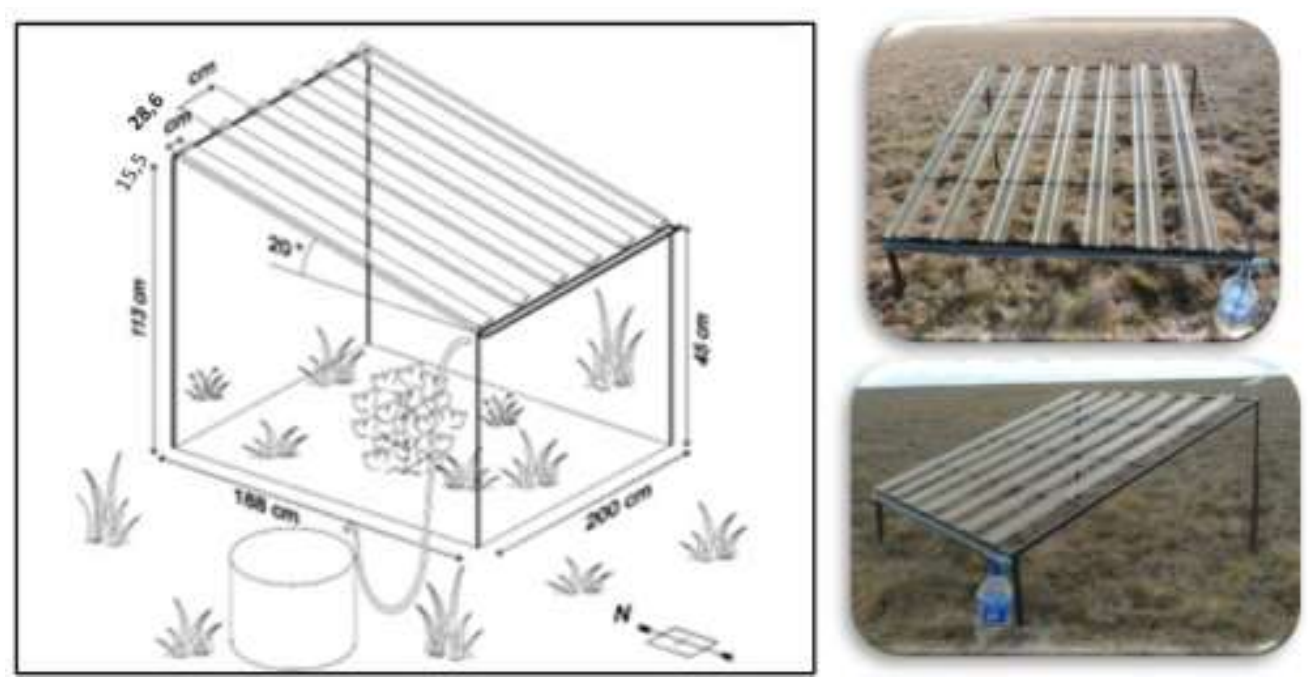

Figura 1: Esquema del Interceptor de precipitaciones (interceptación del 54\%) y fotos de las estructuras instaladas en el campo Experimental Potrok Aike del INTA en la Estepa Magallánica Seca, para lograr el tratamiento de extrema sequía en el marco del ensayo DroughNet. 


\section{Medición de carbono en biomasa microbiana}

En cada una de las parcelas se tomaron tres muestras compuestas por cinco submuestras en los primeros cinco $\mathrm{cm}$ de profundidad con un tubo de PVC al inicio del otoño (abril). Las muestras colectadas fueron preservadas en heladera $\left(4^{\circ} \mathrm{C}\right)$ hasta su posterior análisis en el laboratorio. Las estimaciones del $\mathrm{C}$ en biomasa microbiana se realizaron mediante el método de fumigación-extracción (Vance et al., 1987). Este método se basa en el aumento de la cantidad de $\mathrm{C}$ extractable con $\mathrm{K}_{2} \mathrm{SO}_{4}$ producto de la fumigación (con respecto a la muestra sin fumigar) proveniente del C lábil liberado a la solución del suelo por la muerte de los microorganismos con los vapores de cloroformo. Previamente a la cuantificación de $\mathrm{C}$ en biomasa microbiana las muestras de suelo se llevaron a temperatura ambiente y contenido hídrico a capacidad de campo, tomando submuestras de 30 gr para determinación de $\%$ de humedad ya que las determinaciones se deben expresar en base a peso seco. Luego se tomaron dos submuestras de $50 \mathrm{~g}$ de peso fresco una para control y otra para fumigar. A su vez, una sub-muestra adicional de $30 \mathrm{~g}$ se tomó para determinar el peso seco. Las muestras control fueron extraídas con $50 \mathrm{ml}$ de $\mathrm{K}_{2} \mathrm{SO}_{4} 0,5 \mathrm{M}$, y agitadas en un agitador horizontal por 1 hora. Las muestras a fumigar se colocaron en un desecador junto con un recipiente conteniendo $30 \mathrm{ml}$ de cloroformo libre de etanol, el cual se llevó a ebullición. Luego se dejó reposar por 24 hs en oscuridad. Finalizado este período, se llevó a cabo la extracción y agitación de la misma forma que con las muestras control. Las muestras serán digeridas con solución sulfocrómica por 30 minutos a $150{ }^{\circ} \mathrm{C}$. La concentración de $\mathrm{C}$ de las muestras digeridas se obtuvo mediante la lectura con un espectrofotómetro. Previamente, se realizó una curva de calibración usando biftalato de potasio como patrón. La conversión de $\mathrm{C}$ a biomasa microbiana se realizó mediante la fórmula:

$\mathrm{C}$ en biomasa microbiana $=(\mathrm{Cf}-\mathrm{Cnf}) / \mathrm{KEC}$ donde: $\mathrm{Cf}=$ Carbono en el extracto fumigado; $\mathrm{Cnf}=$ Carbono en el extracto no fumigado $\mathrm{KEC}=$ constante de eficiencia de la fumigación $=$ 0,45 (Jenkinson and Ladd, 1981).

\section{Análisis estadísticos}

Los datos fueron analizados mediante un ANOVA factorial con el programa Infostat 2.0, donde los factores han sido los diferentes contenidos de humedad en el suelo: sequía, riego y control. En caso de encontrar diferencias significativas estas fueron separadas mediante el test de Tukey a un nivel de significancia de $\mathrm{p}<0,05$.

\section{RESULTADOS}

El contenido de carbono en biomasa microbiana fue de 466,15 $( \pm 27,2) \mu \mathrm{g}$ de $\mathrm{C} / \mathrm{g}$ suelo seco para el tratamiento control, mientras que el tratamiento de sequía fue de $394,08( \pm 29,8) \mu \mathrm{g}$ de $\mathrm{C} / \mathrm{g}$ suelo seco y para el de riego $462( \pm 29,6) \mu \mathrm{g}$ de $\mathrm{C} / \mathrm{g}$ suelo seco (Figura 2). No se encontraron diferencias significativas $(\mathrm{p}=0,0552)$ entre los tratamientos para este primer año de medición. 


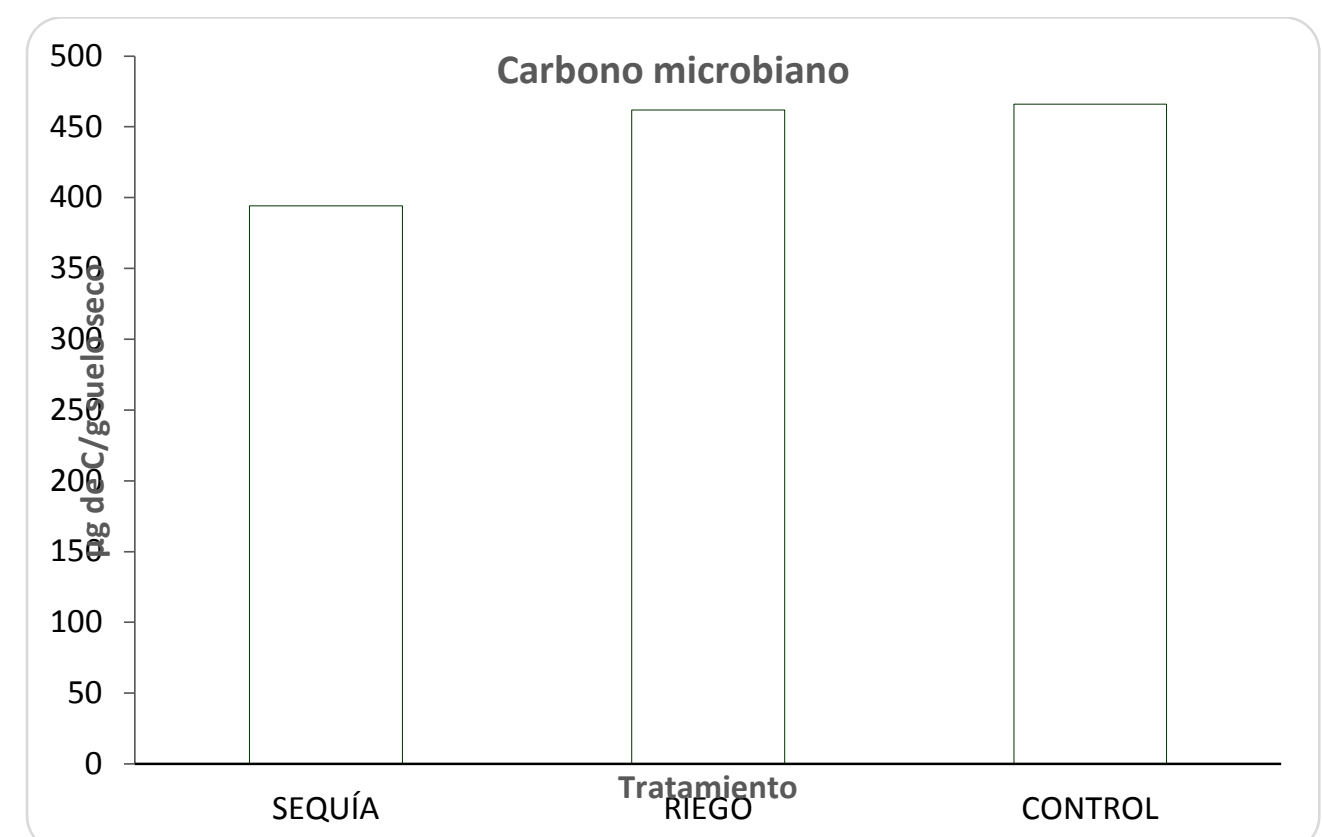

Figura 2. Se muestran los resultados de carbono microbiano para el tratamiento sequía que consistió en una reducción del 54\% en las precipitaciones; control y riego, al cual se le aplicó un aumento del $54 \%$ con respecto a la precipitación media anual de la zona.

\section{DISCUSIÓN}

Los resultados del estudio no plasmaron diferencias estadísticas significativas entre los distintos tratamientos. Sin embargo, en el suelo sometido a sequía se evidenció una tendencia a una disminución en el carbono contenido en la biomasa microbiana. Los valores de C-BM encontrados en este estudio son similares a los informados por Liu et al (2012) en China, en una estepa típica (335 mm anual) y una estepa desértica ( $280 \mathrm{~mm}$ anual), en donde obtuvieron valores de $325 \mu \mathrm{g}$ de $\mathrm{C} / \mathrm{g}$ suelo seco y $475 \mu \mathrm{g}$ de $\mathrm{C} / \mathrm{g}$ suelo seco respectivamente.

Otros estudios en zonas con mayor precipitación, como la región oriental de la amazona en Brasil, con precipitaciones entre 2000-2500 mm anual, Turbay L. et al (2015) obtuvieron valores superiores de CBM (644 $\mu \mathrm{g}$ de $\mathrm{C} / \mathrm{g}$ suelo seco), lo cual podría indicar que microorganismos se ven beneficiados por la buena disponibilidad de humedad en el suelo.

Por otro lado, los suelos sometidos a riego y a tratamiento control arrojaron mayores valores en comparación a la sequía, lo que podría sugerir que a mayor disponibilidad de agua, mayor C-BM. Sin embargo, esto no necesariamente ocurre siempre, en estudios realizados por Fernandez et al (2017) en la región semiárida pampeana Argentina y Albanesi et al (2003) en la región chaqueña Argentina para suelos con vegetación natural los valores de C-MB fueron de $162 \mu \mathrm{g}$ de $\mathrm{C} / \mathrm{g}$ suelo seco y $325,52 \mu \mathrm{g}$ de $\mathrm{C} / \mathrm{g}$ suelo seco respectivamente, valores inferiores incluso con respecto a los arrojados en el tratamiento sequía llevado adelante en el presente trabajo; en zonas del país que tienen aproximadamente el doble de precipitación anual $(600 \mathrm{~mm})$.

\section{CONCLUSIÓN}

Los resultados obtenidos en este estudio indican que tras un año de medición, no se encontraron diferencias significativas en el C- BM entre los distintos niveles de humedad en suelos de estepa magallánica seca en Santa Cruz. Sin embargo, se observó una tendencia de 
disminución en el C- $\mathrm{BM}$ en el tratamiento sequía. Es importante continuar con las mediciones a fin de verificar si estas diferencias persisten, se agrandan o se diluyen con el tiempo.

\section{AGRADECIMIENTOS}

A mis profesores y directores de beca Dr. Peri Pablo y Dra. Gargaglione Verónica, a mi hermano Ing. Toledo Santiago. Ellos me acompañaron durante el período de beca brindándome su acompañamiento, conocimiento y paciencia para poder cumplir con los objetivos propuestos en este trabajo y lograr familiarizarme con el mundo de la investigación hasta ahora desconocido para mí.

A Mónica Bravo, técnica del laboratorio de química de la UNPA-UARG, que en repetidas ocasiones resolvió inconvenientes que se nos presentaron durante las tareas de laboratorio.

A los referentes de la EEA del INTA RIO GALLEGOS, que me permitieron desempeñar mis tareas del presente trabajo en su laboratorio durante los últimos meses de la beca.

\section{BIBLIOGRAFÍA}

ADU J. K., OADES J. M. (1978). Physical factors influencing decomposition of organic materials in soil aggregates. Soil Biology and Biochemistry. 10(2): 109-115. https://doi.org/10.1016/0038-0717(78)90080-9

AERTS, R. (1997). Climate, leaf litter chemistry and leaf litter decomposition in terrestrial ecosystems: a triangular relationship. Oikos 79: 439-449. https://doi.org/10.2307/3546886

ALBANESI, A.; A. ANRIQUEZ Y A. POLO SÁNCHEZ (2003). Efectos de la agricultura convencional sobre algunas formas del $\mathrm{C}$ en una toposecuencia de la Región Chaqueña, Argentina.

ANDERSON T. H., DOMSCH K. H. (1986). Carbon assimilation and microbial activity in soil. Z. Pflanzenernaehr. Bodenk. 149: 457-468. https://doi.org/10.1002/jpln.19861490409

BENDING G., PUTLAND C., RAYNS F. (2000). Changes in microbial community metabolism and labile organic matter fractions as early indicators of the impact of management on soil biological quality. Biol. Fertil. Soils. 31: 78-84. https://doi.org/10.1007/s003740050627

BROCK T. D., SMITH D. W., MADIGAN, M. T. (1984). Biology of microorganisms, fourth ed., Prentice-Hall, Englewood Cliffs pp. 847.

BROOKES P.C. (1995). The use of microbial parameters in monitoring soil pollution by heavy metals. Biol. Fertil. Soils 19: 269-279. https://doi.org/10.1007/BF00336094

CAMPBELL, C.A., BIEDERBECK, V.O., ZENTNER, R.P., LAFOND, G.P., (1991). Effect of crop rotations and cultural practices on soil organic matter, microbial biomass and respiration in a thin Black Chernozem. Can. J. Soil Sci. 71, 363-376. https://doi.org/10.4141/cjss91-035

CARMONA M. R., AGUILERA M., PÉREZ C. A., SEREY I. (2006). Actividad respiratoria en el horizonte orgánico de suelos de ecosistemas forestales del centro y sur de Chile. Gayana. Botánica. 63(1): 1-12. https://doi.org/10.4067/S0717-66432006000100001 
CARTER, M.R., RENNIE, D.A., (1982). Changes in soil quality under no-tillage farming systems: distribution of microbial biomass and mineralizable $\mathrm{C}$ and $\mathrm{N}$ potential. Can. J. Soil Sci. 62, 587-597. https://doi.org/10.4141/cjss82-066

CHAPIN F. S., MATSON P. A., MOONEY H. A. (2002). Principles of terrestrial ecosystem ecology. Springer-Verlag, New York. 436 pp.

COCHRAN V. L., ELLIOTT L. F., LEWIS C. E. (1989). Soil microbial biomass and enzyme activity in subarctic agricultural and forest soils. Biology and Fertility of Soils, 7(4): 283-288. https://doi.org/10.1007/BF00257821

COUTEAUX, M. M., P. BOTTER, BERG, B. (1995). Litter decomposition, climate and litter quality. Trends Ecol Evol10:63-66. https://doi.org/10.1016/S0169-5347(00)88978-8

EDGERTON D. L., HARRIS J. A., BIRCH P., BULLOCK P. (1995). Linear relationship between aggregate stability and microbial biomass in three restored soils. Soil Biology and Biochemistry, 27(11): 1499-1501. https://doi.org/10.1016/0038-0717(95)00076-Q

FERNANDEZ R., FREIZER I., RORIG M., QUIROGA A., NOELLEMEYER E. (2017). Evaluación de indicadores biológicos en suelos de la región semiárida pampeana central. https://doi.org/10.25260/EA.18.28.1.0.555

FIERER N., SCHIMEL J. P. (2003). A proposed mechanism for the pulse in carbon dioxide production commonly observed following the rapid rewetting of a dry soil. Soil Science Society of America Journal, 67(3): 798-805. https://doi.org/10.2136/sssaj2003.0798

FRANZLUEBBERS, A.J., HONS, F.M., ZUBERER, D.A., (1994). Long-term changes in soil carbon and nitrogen pools in wheat Management systems. Soil Sci. Soc. Am. J. 58, 1639-1645. https://doi.org/10.2136/sssaj1994.03615995005800060009x

GARCÍA IZQUIERDO C., GIL SOTRES F., HERNÁNDEZ FERNÁNDEZ T., TRASAR CEPEDA C. (2003). Técnicas de análisis de parámetros bioquímicos en suelos: medida de actividades enzimáticas y biomasa microbiana. Ediciones Mundi-Prensa. España.

GUPTA S. R., SINGH J. S. (1981). Soil respiration in a tropical grassland. Soil Biology and Biochemistry. 13(4): 261-268. https://doi.org/10.1016/0038-0717(81)90060-2

HOUGHTON J. T., DING Y. D. J. G., GRIGGS D. J., NOGUER M., VAN DER LINDEN P. J., DAI X., JOHNSON C. A. (2001). Climate change 2001: the scientific basis.

JENKINSON D. S. (1988). Determination of microbial biomass carbon and nitrogen in soil. In: J.R. Wilson (Editor). Advances in nitrogen cycling in agricultural systems. CAB International.

JENSEN K. D., BEIER C., MICHELSEN A., EMMETT B. A. (2003). Effects of experimental drought on microbial processes in two temperate heathlands at contrasting water conditions. Applied Soil Ecology. 24(2): 165-176. https://doi.org/10.1016/S0929-1393(03)00091-X

KANDELER E., BÖHM K. E. (1996). Temporal dynamics of microbial biomass, xylanase activity, $\mathrm{N}$-mineralisation and potential nitrification in different tillage systems. Applied Soil Ecology. 4(3): 181-191. https://doi.org/10.1016/S0929-1393(96)00117-5

KREPS G., MARTÍNEZ PASTUR G., PERI P. L. (2012). Cambio climático en Patagonia Sur: Escenarios futuros en el manejo de los recursos naturales.Ediciones INTA, Buenos Aires.

LANDGRAF D., KLOSE S. (2002). Mobile and readily available C and N fractions and their relationship to microbial biomass and selected enzyme activities in a sandy soil under different management systems. J. Plant Nutr. Soil Sci. 165: 9-16. https://doi.org/10.1002/1522-2624(200202)165:1<9::AID-JPLN9>3.0.CO;2-O 
LAVELLE P., BLANCHART E., MARTIN A., MARTIN S., SPAIN A., TOUTAIN F., BAROIS I., SCHAEFER R. (1993). A hierarchical model for decomposition in terrestrial ecosystems: application to soils of the humid tropics. Biotropica 25: 130150. https://doi.org/10.2307/2389178

LIU NAN, YINGJUN ZHANG*, SHUJUAN CHANG, HAIMING KAN, LIJUN LIN (2012). Impact of Grazing on Soil Carbon and Microbial Biomass in Typical Steppe and Desert Steppe of Inner Mongolia. https://doi.org/10.1371/journal.pone.0036434

MOGOLLÓN J.P., TORRES D., MARTÍNEZ A. (2010). Cambios en algunas propiedades biológicas del suelo según el uso de la tierra en el sector el cebollal, estado falcon, Venezuela. Bioagro 22(3): 217-222. 2010.

PAUL E. A., CLARK F. E. (1989). Soil microbiology and biochemistry. Academic, San Diego. https://doi.org/10.1016/B978-0-12-546805-3.50004-7

PERI, P. L. (2010). Sistemas silvopastoriles en bosques de Nothofagus antarctica: revisión del conocimiento actual en Patagonia Sur, Argentina. Actas I Congreso Internacional Agroforestal Patagónico, 15 pp. Coyhaique, Chile, 27 al 29 de Octubre de 2010.

RAGHUBANSHI A. S. (1994). Effect of bamboo harvest on dynamics of nutrient pools, N mineralization, and microbial biomass in soil. Biology and fertility of soils. 18(2): 137-142. https://doi.org/10.1007/BF00336460

SINGH J. S., RAGHUBANSHI A. S., SINGH R. S., SRIVASTAVA S. C. (1989). Microbial biomass acts as a source of plant nutrients in dry tropical forest and savanna. Nature, 338(6215), 499-500. https://doi.org/10.1038/338499a0

SWIFT, M. J., Heal, O. W., Anderson J. M. (1979). Decomposition in terrestrial ecosystems. Studies in Ecology 5. Univ. California Press. Berkeley y Los Ángeles.

TRASAR M. C., LEIRÓS M. C., GIL F. (2000). Biochemical properties of acid soils under climax vegetation (Atlantic oakwood) in an area of the European temperate-humid zone (Galicia, NW Spain): specific parameters. Soil Biology \& Biochemistry 32: 747755. https://doi.org/10.1016/S0038-0717(99)00196-0

VAZQUEZ, E. R, Dávila, D. Z. (2008). Efecto de la humedad, temperatura y pH del suelo en la actividad microbiana a nivel de laboratorio. Ecología Aplicada (7)1,2. https://doi.org/10.21704/rea.v7i1-2.367

VISSER, S., PARKINSON, D., (1992). Soil biological criteria as indicators of soil quality: soil microorganisms. Am. J. Alt. Agric. 7(1-2), 33-37. https://doi.org/10.1017/S0889189300004434

WARDLE D. A. (1998). Controls of temporal variability of the soil microbial biomass: a global-scale synthesis. Soil Biology and Biochemistry. 30(13): 1627-1637. https://doi.org/10.1016/S0038-0717(97)00201-0 